\title{
The Drosophila Caspase DRONC Cleaves following Glutamate or Aspartate and Is Regulated by DIAP1, HID, and GRIM*
}

Received for publication, January 31, 2000, and in revised form, May 15, 2000

Published, JBC Papers in Press, May 23, 2000, DOI 10.1074/jbc.M000869200

\author{
Christine J. Hawkins $\$$, Soon Ji Yoołn, Erin P. Peterson|, Susan L. Wang, Stephanie Y. Vernooy, \\ and Bruce A. Hay** \\ From the Division of Biology, MC 156-29, Pasadena, California 91125
}

\begin{abstract}
The caspase family of cysteine proteases plays important roles in bringing about apoptotic cell death. All caspases studied to date cleave substrates $\mathrm{COOH}-$ terminal to an aspartate. Here we show that the Drosophila caspase DRONC cleaves COOH-terminal to glutamate as well as aspartate. DRONC autoprocesses itself following a glutamate residue, but processes a second caspase, drICE, following an aspartate. DRONC prefers tetrapeptide substrates in which aliphatic amino acids are present at the $\mathrm{P} 2$ position, and the $\mathrm{P} 1$ residue can be either aspartate or glutamate. Expression of a dominant negative form of DRONC blocks cell death induced by the Drosophila cell death activators reaper, hid, and grim, and DRONC overexpression in flies promotes cell death. Furthermore, the Drosophila cell death inhibitor DIAP1 inhibits DRONC activity in yeast, and DIAP1's ability to inhibit DRONC-dependent yeast cell death is suppressed by HID and GRIM. These observations suggest that DRONC acts to promote cell death. However, DRONC activity is not suppressed by the caspase inhibitor and cell death suppressor baculovirus p35. We discuss possible models for DRONC function as a cell death inhibitor.
\end{abstract}

Programmed cell death, or apoptosis, is a process by which organisms remove unwanted or damaged cells during development and in the adult (reviewed in Ref. 1). Central components of this process are a family of cysteine proteases known as caspases (2). Caspases are translated as inactive precursors that are cleaved to generate proteolytically active enzymes. Caspase processing involves one or more cleavages $\mathrm{COOH}$ terminal to the active site cysteine to produce large and small subunits. An $\mathrm{NH}_{2}$-terminal prodomain is also often removed. Studies of the crystal structures of caspases show that large and small subunits from two precursor molecules assemble to form an active heterotetramer (3-6). Caspases described to

* This work was supported in part by grants from the Burroughs Wellcome Fund (New Investigator awards in the Pharmacological Sciences), the Ellison Medical Foundation, and National Institutes of Health Grant GM057422-01 (to B. A. H.). The costs of publication of this article were defrayed in part by the payment of page charges. This article must therefore be hereby marked "advertisement" in accordance with 18 U.S.C. Section 1734 solely to indicate this fact.

$\neq$ Contributed equally to the results of this work.

$\S$ Supported by a Human Frontiers postdoctoral fellowship. Current address: Dept. of Haematology and Oncology, Royal Children's Hospital, Flemington Road, Parkville, VIC 3052, Australia.

ๆ Supported by a Jane Coffin Child postdoctoral fellowship.

\| Current address: Dept. of Enzymology, Merck Research Laboratories, Rahway, NJ 07065.

** Searle Scholar. To whom correspondence should be addressed. Tel.: 626-395-3399: Fax: 626-449-0756; E-mail: haybruce@its. caltech.edu. date all cleave their substrates following aspartate residues (7-11). Importantly, the sites at which caspase zymogens are cleaved to generate active tetramers often correspond to consensus caspase target sites. This has suggested that caspases can function in a cascade in which initiator caspases, activated by upstream death signals, cleave and activate a set of executioner caspases that carry out proteolytic cleavages of cellular proteins $(12,13)$.

Seven caspases, DCP-1 (14), drICE (15), DCP-2/DREDD (16, 17), DRONC (18), DECAY (19), and two caspases predicted on the basis of genomic sequence (20) have been identified in Drosophila. Evidence that caspases are important for cell death in Drosophila comes from several sets of observations. Expression of the baculovirus caspase inhibitor p35 or the Drosophila caspase inhibitor DIAP1 blocks cell death in the fly in a number of different contexts (reviewed in Ref. 21), including normally occurring cell death and death induced by overexpression of the cell death activators reaper ( $r p r)$, head involution defective (hid), and grim (22-26). Dominant negative forms of DCP-2/ DREDD (27) and DRONC (28) (this work) inhibit rpr-, hid-, and grim-dependent cell death. Mutations in $d c p-1$ (29), the Drosophila homolog of the caspase-activating adaptor Apaf-1 (27, 30,31 ), or heterozygosity for deficiencies that remove the $d c p$ $2 /$ dredd (17) or dronc loci (28), suppress apoptosis in specific contexts. Also, immunodepletion of drICE prevents apoptotic events in cell extracts (32). Finally, mutants that eliminate the function of a Drosophila caspase inhibitor, DIAP1, result in massive cell death (33-35), which is associated with an increase in caspase activity (33).

How do the Drosophila caspases function to bring about cell death in the fly? DCP-1, drICE, DECAY, and one caspase predicted by genomic sequence (daydream; GenBank ${ }^{\mathrm{TM}}$ accession number AF281077) have short prodomains characteristic of executioner caspases. In contrast, DCP-2/DREDD and DRONC have large $\mathrm{NH}_{2}$-terminal prodomains with homology to mammalian death effector and caspase recruitment domains, respectively. A second caspase predicted by genomic sequence (dream; GenBank ${ }^{\mathrm{TM}}$ accession number AF275814) has a long prodomain that lacks homology with any known death regulators. Death effector and caspase recruitment domains in caspases are thought to mediate their recruitment to death signal-dependent complexes in which activation occurs in response to oligomerization (reviewed in Ref. 36). Thus DCP2/DREDD and DRONC may act as initiator caspases in apoptotic signaling.

In most caspases the catalytic site cysteine $(\mathrm{C})$ is present in the pentapeptide sequence $\mathrm{QAC}(\mathrm{R} / \mathrm{Q} / \mathrm{G})(\mathrm{G} / \mathrm{E})$, in which the $\mathrm{QAC}$ motif is invariant. DRONC is unique among caspases in that the sequence surrounding the active site is PFCRG (Fig. $1 A$ ) (18). Caspase crystal structures indicate that the glutamine at the first position of the canonical caspase pentapeptide 
QACRG is part of the substrate binding pocket. The fact that DRONC has a proline at this position suggests that it has a novel substrate specificity. Several Caenorhabditis elegans caspases, CSP-1a and CSP-2a, with pentapeptide sequences that differ in the first two pentapeptide positions, SACRG and VCCRG, respectively, have also been described (37).

Here we show that, unique among caspases characterized to date, DRONC cleaves tetrapeptide and protein substrates COOH-terminal to glutamate as well as aspartate residues. A role for DRONC as a cell death caspase is suggested by the observations that expression of a dominant negative form of DRONC blocks cell death, that DRONC expression induces cell death, and that DRONC interacts with other Drosophila cell death regulators, including DIAP1, drICE, hid, and grim. Interestingly, however, DRONC is not inhibited by baculovirus p35, which inhibits cell death in flies.

\section{EXPERIMENTAL PROCEDURES}

PS-SCL Library Analysis-The substrate specificity of recombinant DRONC was tested using positional scanning synthetic combinatorial libraries, as described previously $(10,38)$.

Recombinant Protein Production, DRONC Microsequencing, and Caspase Activity Assays-The DRONC coding region was amplified by polymerase chain reaction and introduced into pET23(a) (Novagen) to produce pDRONC-His ${ }_{6}$. This plasmid was used to prepare active DRONC from Escherichia coli as described for DCP- $1^{31}-\mathrm{His}_{6}$ (39). The subunits were resolved on a $15 \%$ SDS-PAGE $^{1}$ gel and transferred to polyvinylidene difluoride (Millipore). The membrane was stained with Coomassie and the smaller band excised. Microsequencing was carried out at the Caltech Protein Microanalytical Laboratory under the direction of Gary M. Hathaway. Fluorogenic peptide cleavage assays were carried out as described (39), using DRONC or DCP-1 at $0.2 \mu \mathrm{M}$ or 0.2 $\mathrm{nM}$ final concentration as specified in the text. AFC-tetrapeptide substrates were purchased from Enzyme Systems (Livermore, CA), including the custom made Ac-TQTE-AFC and AC-TQTD-AFC. Bacterially produced DCP-1-His ${ }_{6}$ and drICE-His ${ }_{6}$ have been described (39).

${ }^{35}$ S-Protein Cleavage Assays-The coding regions for DRONC, drICE, and DCP-1 were cloned from the yeast expression constructs into Bluescript KS+ (Stratagene). The coding regions of mutant versions of these proteins were generated using polymerase chain reaction and the QuickChange site-directed mutagenesis kit (Stratagene). Sequencing verified the existence of the desired mutation and the absence of other polymerase chain reaction-generated mutations. A transcription-coupled rabbit reticulocyte translation system (TNT, Promega) with Redivue $\left[{ }^{35}\right.$ S $]$ methionine (Amersham Pharmacia Biotech) was used to generate ${ }^{35} \mathrm{~S}$-labeled proteins. $2 \mu \mathrm{l}$ of each ${ }^{35} \mathrm{~S}$-labeled product was incubated with $2 \mu \mathrm{M}$ of active caspase or buffer alone in caspase activity buffer (39) in a total $10-\mu \mathrm{l}$ volume. The products were resolved by $15 \%$ SDS-PAGE, the proteins transferred to Hybond ECL membrane (Amersham Pharmacia Biotech). Bio-Max MR-1 film (KODAK) was used to visualize the labeled fragments.

Characterization of DRONC Activity-The concentration of active DRONC was determined by active site titration using the active site inhibitor carbobenzoxy-VAD-fluoromethyl ketone (z-VAD-fmk), as described in Ref. 40. The activity of DRONC was measured in triplicate for each substrate using continuous fluorometric assays as described in Ref. 33. Appropriate dilutions of enzyme were added to reaction mixtures containing substrate and caspase activity buffer (33) in a total volume of $100 \mu \mathrm{l} . k_{\text {cat }} / K_{m}$ values were calculated as described in Ref. 41.

Yeast Constructs and Assays-Yeast expression plasmids for galactose inducible expression of DCP-1, DIAP1, DIAP2, and P35 have been previously described (39). pCUP-DIAP1, for copper inducible expression of DIAP1, pGALL-RPR, pGALL-GRIM, and pGALL-HID have been previously described (33). The coding region of DRONC was amplified from a clone (LD12627) obtained from the Berkley Drosophila Genome project, and cloned into pGALL-(LEU2) (39) to produce pGALLDRONC. A DRONC active site mutant, DRONC ${ }^{\mathrm{C} 318 \mathrm{~S}}$, was generated by polymerase chain reaction using a mutagenic oligonucleotide encompassing the internal SacII site of DRONC, and cloned to replace the corresponding section of wild-type DRONC in pGALL-(LEU2), generating pGALL-DRONC ${ }^{\mathrm{C} 318 \mathrm{~S}}$. A section of human PARP encoding amino

${ }^{1}$ The abbreviations used are: PAGE, polyacrylamide gel electrophoresis; PARP, poly(ADP-ribosyl)transferase. acids 1-337, spanning the caspase cleavage site, was amplified from a HeLa cell Matchmaker library (CLONTECH) and cloned into pADH(TRP1) (33) in front of a Myc epitope tag (encoding MEQKLISEEDLAS), to generate pADH-mycPARP ${ }^{337}$. A fragment encoding p35 was excised from pEF-p35 (42) and cloned into pADH-(TRP1) (33) to give pADH-p35.

W303 $\alpha$ yeast were transformed as described previously (39). For survival assays, transformants were grown in selective liquid medium to saturation, then pelleted, and resuspended in TE to $A_{600}=0.02$. Five-fold dilutions were prepared, and $2-\mu \mathrm{l}$ drops of each solution were spotted onto selective plates with galactose and 0,10 , or $100 \mu \mathrm{M} \mathrm{CuSO}_{4}$ as specified. For Western blotting to analyze p35 and PARP cleavage, transformants were grown in glucose-containing selective medium to stationary phase, washed in TE and grown in YP medium with galactose for $8 \mathrm{~h}$. Yeast were pelleted, lysed by 2 cycles of boiling, and vortexing with glass beads in cracking buffer ( $8 \mathrm{~m}$ urea, $5 \%$ SDS, $40 \mathrm{mM}$ Tris, pH 6.8, $0.1 \mathrm{~mm}$ EDTA, $1 \% \beta$-mercaptoethanol, $0.4 \mathrm{mg} / \mathrm{ml}$ bromphenol blue). Samples were subjected to $12 \%$ SDS-PAGE, transferred to Hybond ECL membrane (Amersham Pharmacia Biotech), and probed with antibodies recognizing the Myc epitope or p35. Washes were followed by incubation with a goat anti-mouse horseradish peroxidaseconjugated secondary antibody (1:2000), followed by detection with ECL (Pierce).

Transgenic Drosophila Genetics and Histology-The coding regions for full-length wild type DRONC, DRONC ${ }^{\mathrm{C} 318 \mathrm{~S}}$, GRIM, and HID were cloned into pGMR (22), and introduced into the Drosophila germline using standard techniques (43), generating GMR-DRONC GMRDRONC ${ }^{\mathrm{C} 318 \mathrm{~S}}$, GMR-grim, and GMR-hid flies, respectively. GMR-rpr (24) and GMR-p35 (22) flies have been described previously. Fixation, embedding and sectioning of adult fly heads, and acridine orange staining were carried out as described in Ref. 44.

\section{RESULTS AND DISCUSSION}

DRONC Cleaves Itself COOH-terminal to a GlutamateSome caspases autocatalytically cleave and activate themselves. To determine if and where DRONC cleaves itself we expressed and purified from $E$. coli a $\mathrm{COOH}$-terminal $\mathrm{His}_{6}{ }^{-}$ tagged version of DRONC. The purified protein consisted of two major bands, presumably consisting of the processed large and small subunits (data not shown). As discussed below, this protein is active as a caspase. To determine the site of cleavage between the large and small subunits, Edman degradation amino-terminal sequencing was performed on the smaller band. The $\mathrm{NH}_{2}$-terminal sequence determined (Fig. $1 A$ ) occurs $\mathrm{COOH}$-terminal to the sequence $\mathrm{TQTE}^{352}$, suggesting that DRONC cleaves itself following a glutamate rather than an aspartate. To test this hypothesis directly we mutated DRONC $\mathrm{TQTE}^{352}$ to $\mathrm{TQTA}^{352}$. ${ }^{35} \mathrm{~S}$-Labeled wild type DRONC and DRONC TQTA $^{\mathrm{E} 352 \mathrm{~A}}$ were generated by in vitro translation and incubated with bacterially produced DRONC or DCP-1. As shown in Fig. $1 B$, DRONC cleaved itself to generate a product corresponding in size to the prodomain and large subunit. This band was not seen when DRONC TQTA ${ }^{\mathrm{E} 352 \mathrm{~A}}$ was the substrate, consistent with the hypothesis that DRONC processes itself following TQTE ${ }^{352}$. DCP-1 (Fig. 1B) and drICE (data not shown) cleaved DRONC at several sites. This cleavage was unaffected by the presence of the TQTA ${ }^{352}$ mutation, suggesting that these caspases cleaved elsewhere in DRONC, perhaps in the DRONC prodomain. To explore the possibility of cleavage within the DRONC prodomain we generated a form of DRONC, DRONC ${ }^{\mathrm{pD} 4 \mathrm{~A}}$, in which the $\mathrm{P} 1$ aspartates of four potential caspase target sites within the prodomain, $\mathrm{DEKD}^{66}$, $\mathrm{ESVD}^{110}, \mathrm{DESD}^{113}$, and DIVD ${ }^{135}$, were changed to alanine. As shown in Fig. $1 B,{ }^{35}$ S-labeled in vitro translated $\mathrm{DRONC}^{\mathrm{pD} 4 \mathrm{~A}}$ was still processed by wild type DRONC, but not by DCP-1. Similar results were obtained with cleavage by drICE (data not shown). Thus DCP-1 and drICE can process DRONC within the prodomain, but not at the large-small subunit boundary. If this processing occurs in vivo it may serve as a point of regulation of DRONC function.

DRONC Cleaves Peptide Substrates Containing a P1 Gluta- 


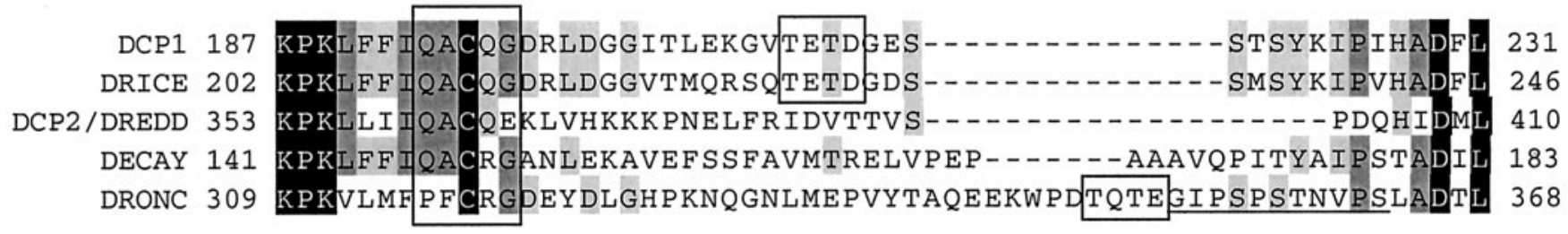

B

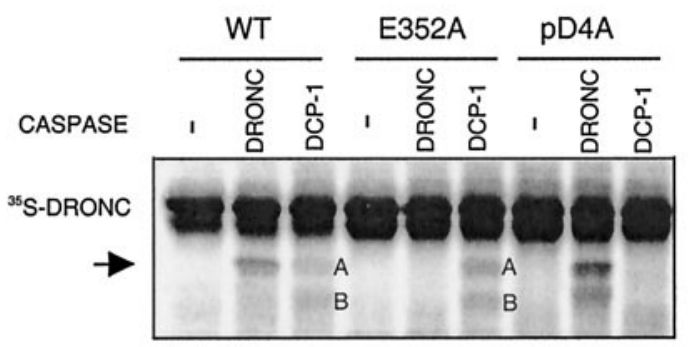

FIG. 1. Cleavage of DRONC by itself and other caspases. Sequence alignments for the five published Drosophila caspases. Residues shared by three proteins are lightly shaded, those shared by four proteins more darkly shaded, and those shared by all caspases outlined in black. The pentapeptide sequence surrounding the caspase active site cysteine and the predicted or demonstrated tetrapeptide cleavage sites in the linker that separates the large and small subunits of the proteins are boxed. The $\mathrm{NH}_{2}$-terminal sequence determined for the DRONC small subunit from protein sequencing is underlined $(A)$. Three ${ }^{35}$ S-labeled in vitro translated versions of DRONC were generated: wild type DRONC (WT), DRONC in which glutamate 352 in the large-small subunit linker was mutated to alanine (E352A), and a version of DRONC in which the P1 aspartates of four potential caspase cleavage sites in the prodomain were mutated to alanine (pD4A). These were incubated alone or with bacterially produced wild type DRONC or DCP-1 as described ("Experimental Procedures") and processed for SDS-PAGE. DRONC processed wild type DRONC to generate a product of the size expected for the prodomain and large subunit (arrow). DRONC TETA ${ }^{352}$ was not processed by DRONC. DCP-1

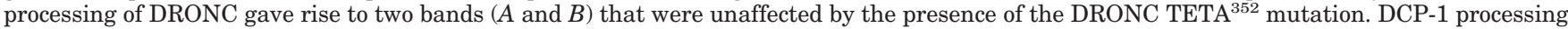
of DRONC was eliminated when four aspartates in the DRONC prodomain were mutated to alanine $(B)$.

mate or Aspartate-Positional scanning synthetic combinatorial libraries (PS-SCL) have been a useful tool to determine cleavage site specificities of other caspases (10). The PS-SCL is composed of three separate sublibraries of 8,000 compounds each. In each sublibrary, one position is defined with one of 20 amino acids (excluding cysteine), while the remaining two positions contain a mixture of amino acids present in approximately equimolar concentrations. Analysis of the three sublibraries (20 samples each) affords a complete understanding of the amino acid preferences in the P2, P3, and P4 positions. We used this approach to characterize DRONC's preferences for given amino acids at each of these positions. The positional scanning synthetic combinatorial libraries available all contain aspartate at the $\mathrm{P} 1$ position. While DRONC cleaves itself after glutamate, it is also able to cleave protein substrates after aspartate (below). Thus we reasoned that the existing aspartate-based libraries would yield useful information about DRONCs cleavage specificity. As shown in Fig. 2A, DRONC shows a strong preference for Thr, Ile, or Val at the P2 position. A wider spectrum of amino acids was tolerated at the P3 and P4 positions. This analysis suggests that TATD constitutes an optimal DRONC P1 aspartate tetrapeptide cleavage site.

The results of the PS-SCL analysis were supported by experiments in which DRONC activity was tested directly with a number of commonly used tetrapeptide activity substrates. DRONC showed highest levels of activity with the tetrapeptides VEID-AMC and IETD-AMC, and somewhat lower levels of activity with DEVD-AMC (Fig. 2B). However, little if any activity was seen with WEHD-AMC or YVAD-AMC, which are predicted to be poor substrates. Fig. $2 B$ also showed that DRONC had higher levels of activity with the pentapeptide
GIETD-AMC than with the tetrapeptide IETD-AMC. This, as well as other observations below, suggests that a P5 residue is important for optimal DRONC activity.

To further characterize DRONCs cleavage preferences we carried out assays in which the cleavage activities of DRONC and DCP-1 were measured for two different peptide substrates: Ac-TQTE-AFC and Ac-DEVD-AFC (Fig. 2C). Ac-TQTE-AFC is derived from the known DRONC autoprocessing site and is also predicted to correspond to a good DRONC cleavage site based on the results obtained from PS-SCL analysis. Ac-DEVD-AFC is a tetrapeptide substrate for caspases generally grouped together as effectors of apoptosis (group II caspases (10)). DRONCs activity is low in absolute terms compared with DCP-1. However, DRONC shows a clear cleavage preference for the Ac-TQTE-AFC substrate over Ac-DEVD-AFC (Fig. 2C). As expected, DCP-1, which has a common variant of the standard caspase active site pentapeptide (QACQG), has a strong preference for the tetrapeptide substrate with a $\mathrm{P} 1$ aspartate, Ac-DEVD-AFC (Fig. 2C) (45). Despite the fact that DRONC shows relatively low levels of activity with tetrapeptide substrates containing a P1 aspartate, DRONC is efficiently inhibited by the broad range tripeptide caspase inhibitor carbobenzoxy-VAD-fluoromethyl ketone (z-VAD-fmk) (data not shown).

We wanted to determine DRONC's P1 specificity with respect to aspartate and glutamate. To do this we synthesized a second tetrapeptide substrate, Ac-TQTD-AFC, that differs from Ac-TQTE-AFC only by the $\mathrm{P} 1$ residue. We used these substrates to measure DRONCs activity $\left(k_{\text {cat }} / K_{m}\right)$, as described under "Experimental Procedures." We calculated a $\left(k_{\text {cat }} / K_{m}\right)$ of 2.73 for TQTE-AFC and a $\left(k_{\text {cat }} / K_{m}\right)$ of 3.36 for TQTD-AFC. Thus DRONC shows only a slight preference for cleavage of 

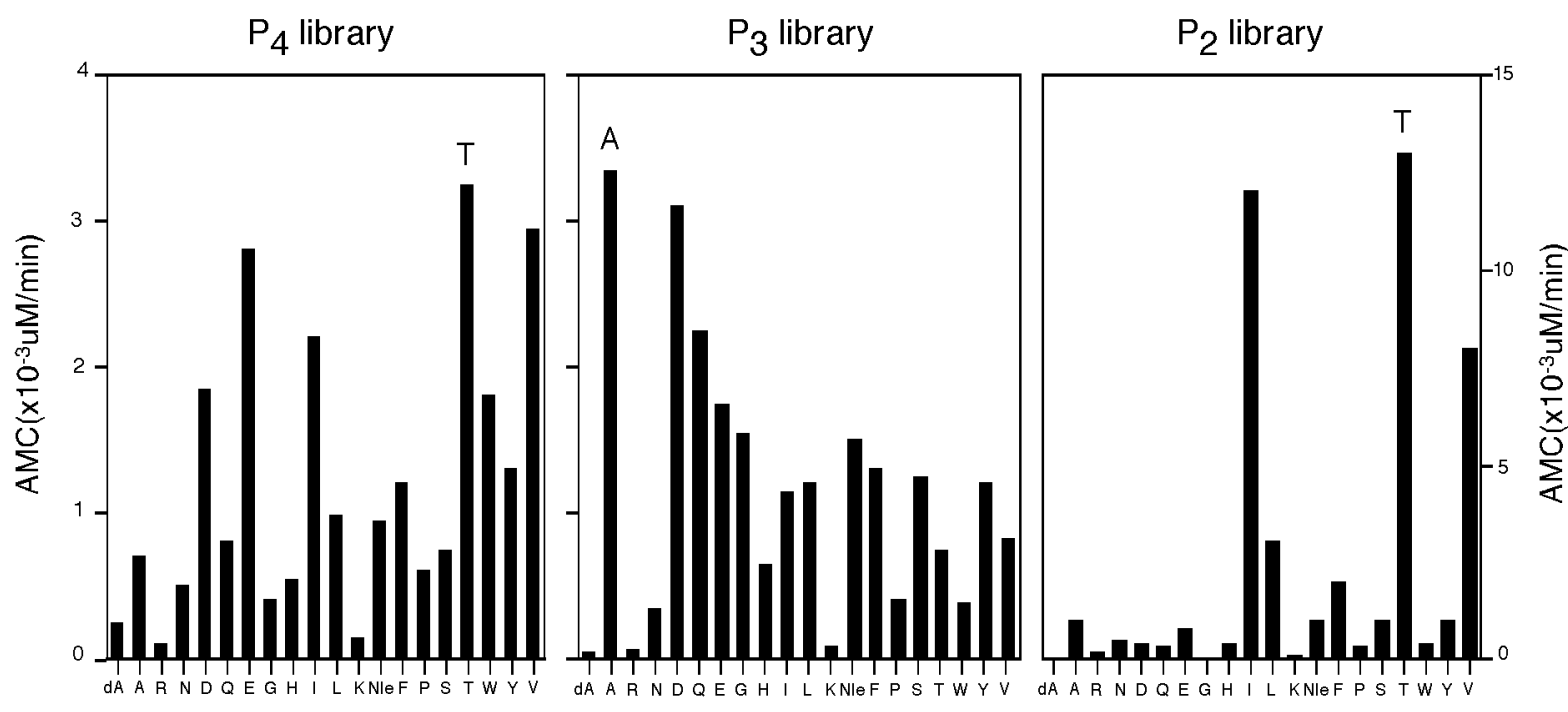

B
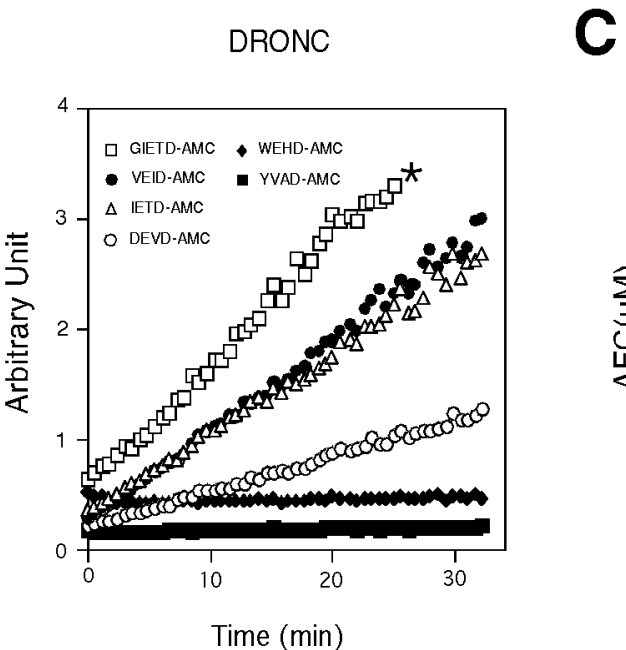

DRONC

DCP-1

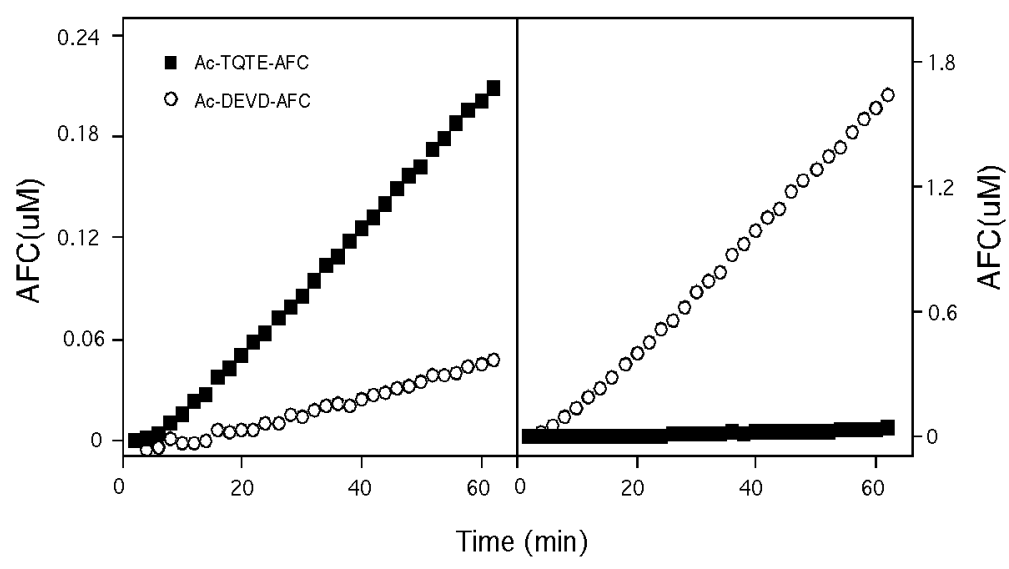

FIG. 2. DRONC cleavage of peptide substrates. Three positional scanning synthetic substrate combinatorial libraries were used to assess the specificity of DRONC for fluorogenic tetrapeptide substrates. In these libraries the P1 amino acid was aspartate, and the P2, P3, or P4 amino acid was varied while pools of all amino acids occupied the other positions. The $y$ axis represents rate of AMC production expressed as micromolar/min. DRONC exhibited marked preference for the aliphatic residues threonine, isoleucine, or valine at the P2 position, but tolerated a wider spectrum of P3 and P4 amino acids $(A)$. DRONC $(0.2 \mu \mathrm{M})$ cleaved the fluorogenic substrates GIETD-AMC, VEID-AMC, IETD-AMC, DEVD-AMC, WEHDAMC, and YVAD-AMC with varying degrees of efficiency. The asterisk $(*)$ present in the GIETD data points indicates the time at which detection saturation occurred $(B)$. DRONC $(0.2 \mu \mathrm{M})$ cleaved Ac-TQTE-AFC $(100 \mu \mathrm{M})$ and Ac-DEVD-AFC $(100 \mu \mathrm{M})$. DCP1 (0.2 nM) cleaved Ac-DEVD-AFC (100 $\mu \mathrm{M})$, but not Ac-TQTE-AFC $(100 \mu \mathrm{M})$. Note the difference in scales for DRONC and DCP-1 cleavage activities $(C)$.

tetrapeptide substrates with a P1 aspartate over those with a $\mathrm{P} 1$ glutamate. DRONC is, however, a particularly poor catalyst of tetrapeptide hydrolysis. The calculated $\left(k_{\text {cat }} / K_{m}\right)$ values for DRONC are roughly 40-180-fold lower than those described for caspase-9, which itself is a very inefficient enzyme in isolation as compared with most other caspases (40). This may reflect the fact that DRONC has an intrinsically low turnover rate or that we have not identified optimal in vitro assay conditions. However, DRONC activity may also be regulated allosterically through interactions with the Drosophila homolog of Apaf-1 (variously known as Dapaf-1 (30), HAC-1 (31), and DARK (27)) in a manner similar to that of mammalian caspase- 9 by Apaf-1 (46). Alternatively, since DRONC shows similar levels of activity to DCP-1 on the protein substrate drICE (below), optimal DRONC cleavage may require additional sequences surrounding the target site.

DRONC and DCP-1 Process drICE COOH-terminal to Aspar- tate Residues-If DRONC is an apical cell death caspase, likely substrates include other Drosophila caspases. drICE is a good candidate to be such a target since immunodepletion experiments show that drICE is required for rpr-dependent apoptotic events in cell extracts (32), and genetic interactions suggest that DRONC contributes to rpr-, hid-, and grim-dependent cell death (see Ref. 28 and below). We generated ${ }^{35} \mathrm{~S}$-labeled in vitro translated drICE and incubated it with bacterially produced DRONC or DCP-1. We found, consistent with the observations of others $(28,45)$, that drICE was efficiently cleaved by DRONC and DCP-1. We observed the generation of a band corresponding in size to that of the mature large subunit, $\mathrm{Ala}^{29}-\mathrm{Asp}^{230}$ (Fig. 3A, band 3). Several other cleavage products were also generated. These correspond to full-length drICE lacking the prodomain, $\mathrm{Ala}^{29}-\mathrm{Val}^{339}$ (band 1), and a fragment comprising the prodomain and large subunit processed at the $\mathrm{COOH}$ terminus of the large-small subunit linker region, 1-Asp ${ }^{230}$ (band 
A

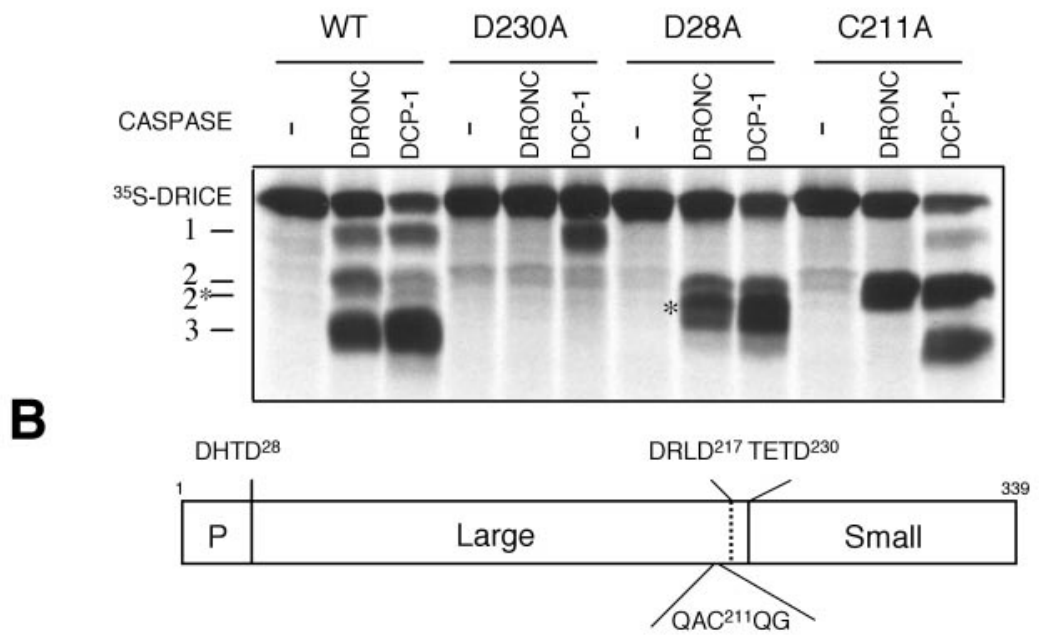

FIG. 3. Processing of drICE by DRONC and DCP-1. Four ${ }^{35}$ S-labeled in vitro translated versions of drICE were generated: wild type (WT), drICE TETA ${ }^{230}$ (D230A), in which the P1 aspartate of a caspase target site in the large-small subunit linker was altered to alanine, drICE ${ }^{\mathrm{D} 28 \mathrm{~A}}$ (D28A), in which the P1 aspartates of the two predicted caspase target sites in the drICE prodomain were changed to alanine, and drICE ${ }^{\mathrm{C} 211 \mathrm{~A}}$ (C211A), in which the drICE active site cysteine was changed to alanine. In vitro translation products were incubated with bacterially produced DRONC or DCP-1 as described ("Experimental Procedures") and processed for SDS-PAGE. DRONC or DCP-1 processing of drICE resulted in the appearance of three bands corresponding to full-length drICE without the prodomain (band 1), the prodomain and the large subunit (band 2), and the fully processed large subunit (band 3). A fourth band $\left(2^{*}\right)$, observed when drICE ${ }^{\mathrm{D} 28 \mathrm{~A}}$ was cleaved by DRONC or DCP-1, may correspond to the prodomain and a version of the large subunit in which separation from the small subunit occurred at DRLD ${ }^{217}(A)$. Schematic showing the location of the drICE subunits, caspase target sites and the active site pentapeptide $(B)$.

2). To show that DRONC and DCP-1 processed drICE at the proposed natural drICE cleavage site between large and small subunits, TETD ${ }^{230}$, we changed this site to TETA ${ }^{230}$. As shown in Fig. $3 A$, DRONC and DCP-1 did not cleave ${ }^{35}$ S-labeled in vitro translated drICE TETA ${ }^{230}$ between the large and small subunit, implying that they both cleave drICE at TETD ${ }^{230}$. These results, taken together with the observed site of DRONC autoprocessing in bacteria and in vitro, and the results of tetrapeptide cleavage experiments, argue that DRONC cleaves following glutamate as well as aspartate. DRONC efficiently processed drICE at QTETD ${ }^{230}$. However, it processed DCP-1 at the equivalent site in the large-small subunit linker, VTETD ${ }^{215}$ (Fig. 1A), very poorly (data not shown). These results are consistent with the possibility, discussed above in the context of DRONCs cleavage of tetrapeptide substrates, that the optimal DRONC peptide substrate is a pentapeptide.

The drICE TETA ${ }^{230}$ mutant was still cleaved by DCP-1 at one position, perhaps at the prodomain-large subunit boundary (Fig. 3). To explore this possibility we altered the P1 aspartate of the proposed prodomain-large subunit boundary caspase target site, $\mathrm{DHTD}^{28}$ (32), to alanine, generating drICE ${ }^{\mathrm{D} 28 \mathrm{~A}}$. DRONC and DCP-1 both processed drICE ${ }^{\mathrm{D} 2 \mathrm{~A}}$ to generate a fragment corresponding in size to the prodomain and large subunit processed at $\mathrm{Asp}^{230}$. A slightly smaller band, probably corresponding to the prodomain and the large subunit processed at the $\mathrm{NH}_{2}$ terminus of the large-small subunit linker, $1-\mathrm{Asp}^{217}$ (band $2^{*}$ ), was also produced. However, no bands corresponding in size to full-length drICE lacking the prodomain or the fully processed large subunit were observed. These observations demonstrate that DCP-1 processes drICE in the prodomain as well as at TETD ${ }^{230}$.

Addition of DRONC to in vitro translated drICE resulted in production of a mature drICE large subunit lacking prodomain sequences, but DRONC was unable to process drICE TETA ${ }^{230}$ within the prodomain (Fig. 3A). These observations suggested that drICE cleaved by DRONC at TETD ${ }^{230}$ was autocatalytically removing its own prodomain. To test this possibility we incubated DRONC and DCP-1 with an in vitro translated ver- sion of drICE, drICE ${ }^{\mathrm{C} 211 \mathrm{~A}}$, in which the active site cysteine was changed to alanine. This caspase should remain inactive following cleavage at $\mathrm{TETD}^{230}$. As shown in Fig. 3A, DRONC cleavage of $\mathrm{drICE}^{\mathrm{C} 211 \mathrm{~A}}$ resulted in the appearance of only a single band corresponding to the prodomain and large subunit. This observation suggests that drICE autocatalytically removes its own prodomain following cleavage between the large and small subunits. Mature drICE ${ }^{\mathrm{C} 211 \mathrm{~A}}$ large subunit was generated in the presence of DCP-1. This further supports the argument that DCP-1 cleaves drICE in the prodomain as well as at the large-small subunit boundary.

What purpose could be served by DRONC having an altered cleavage specificity? One possibility is simply that DRONC has unique targets other than itself, and that a different target site preference is required for cleavage of these substrates. DRONC's novel cleavage site specificity, in conjunction with the sequence of the linker between the large and small subunits, may also provide a mechanism for limiting DRONC's ability to become activated by other caspase cascades. DCP-1 or drICE did not process DRONC to any significant extent at the large-small subunit boundary. This is not surprising because there are only two aspartates in the linker region between the large and small subunits, $\mathrm{DEYD}^{324}$ and KWPD ${ }^{348}$. Based on positional scanning synthetic combinatorial library analysis of tetrapeptide substrates, these sequences are predicted to be very poor substrates for all known mammalian caspases and DCP-1 $(10,11,45)$. We cannot rule out the possibility that processing of DRONC by unknown proteases occurs at these sites in vivo. However, because DRONC was able to process itself in the linker region at TQTE ${ }^{352}$, but other tested caspases were not, it seems reasonable that DRONCs altered cleavage specificity, coupled with the lack of good target sites for other caspases in the large-small subunit linker region, may serve at least in part to make activation of DRONC more strictly DRONCdependent. This may provide a mechanism for limiting crosstalk between other caspase cascades and pathways activated by DRONC.

Expression of an Active Site Mutant of DRONC Suppresses 


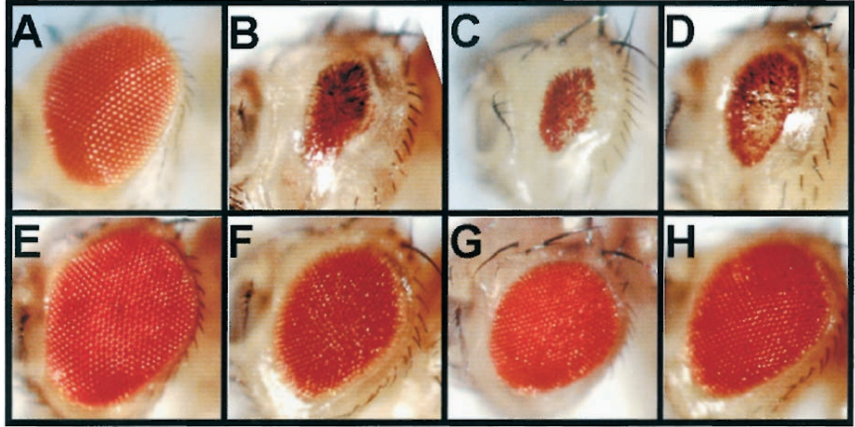

FIG. 4. DRONC ${ }^{\text {C318S }}$ suppresses rpr-, hid-, and grim-dependent cell death in the fly eye. The following genotypes are shown: wild type $(A)$; GMR-hid/+ (B); GMR-rpr/+ (C); GMR-grim/+ (D); GMRDRONCC318S/+ $(E)$; GMR-DRONC $318 \mathrm{~S} /+;$ GMR-hid/+ (F); GMR-

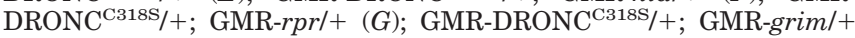
$(H)$. Expression of GMR-hid, GMR-rpr, or GMR-grim resulted in a small eye phenotype $(B-D)$ that was suppressed by coexpression of GMR$\operatorname{DRONC}^{\mathrm{C} 318 \mathrm{~S}}(F-H)$

Cell Death in the Fly-Mutations that remove DRONC are not available. Therefore, to examine a possible role for DRONC as a cell death effector we generated a form of DRONC, $\mathrm{DRONC}^{\mathrm{C} 318 \mathrm{~S}}$, in which the active site cysteine was altered to serine. Expression of similar forms of other caspases results in a suppression of caspase activity and caspase-dependent cell death. This may occur as a result of interaction of DRONC ${ }^{\mathrm{C} 318 \mathrm{~S}}$ with the Drosophila homolog of the caspase-activating protein Apaf-1 (30), thus preventing the Drosophila Apaf-1 from binding to wild type DRONC and promoting its activation in a manner similar to that described for mammalian Apaf-1 and caspase-9 (46-48). We generated transgenic Drosophila in which DRONC ${ }^{\mathrm{C} 318 \mathrm{~S}}$ was expressed under the control of a promoter, known as GMR, that drives transgene expression specifically in the developing fly eye (22). The eyes of these flies, known as GMR-DRONC ${ }^{\text {C318S }}$ flies (Fig. $4 E$ ), appeared similar to those of wild type flies (Fig. 4A). To assay the ability of $\mathrm{DRONC}^{\mathrm{C} 318 \mathrm{~S}}$ to block cell death, GMR-DRONC ${ }^{\mathrm{C} 318 \mathrm{~S}}$ flies were crossed to flies overexpressing rpr (GMR-rpr), hid (GMR-hid), or grim (GMR-grim) under the control of the same promoter. As shown in Fig. 4, $B-D$, and previously (23-26), GMR-driven expression of $r p r$, hid, or grim resulted in a small eye phenotype due to activation of caspase-dependent cell death. However, flies coexpressing GMR-DRONC ${ }^{\mathrm{C} 318 \mathrm{~S}}$ and one of the cell death activators showed a dramatic suppression of the small eye phenotype, indicating that cell death had been suppressed (Fig. 4, F-H). We cannot rule out the possibility that this suppression is a result of DRONC ${ }^{\mathrm{C} 318 \mathrm{~S}}$ forming nonproductive interactions with the Drosophila Apaf-1 that block its ability to activate other long prodomain caspases such as DCP-2/DREDD (27). However, these possibilities notwithstanding, our results and those of Meier et al. (28) suggest that DRONC activity is important for bringing about rpr-, hid-, and grim-dependent cell death.

Cell Death Induced by DRONC Expression Is Suppressed by DIAP1, but Not by Baculovirus p35-We also generated flies that expressed full-length wild type DRONC under GMR control. While phenotypes displayed by individuals within a line were similar, different lines displayed eyes with various degrees of eye disruption, presumably owing to genomic position effects on the expression level of the transgene (49). By manipulating the number of copies of the GMR-DRONC transgene in animals we inferred a phenotypic series in which low levels of DRONC expression (GMR-DRONCW ${ }^{\mathrm{W}}$ flies) resulted in no outward phenotype (data not shown), while higher levels of expression (GMR-DRONC ${ }^{\mathrm{M}}$ flies) resulted in cell death late in retinal development. These flies had eyes that were normal in

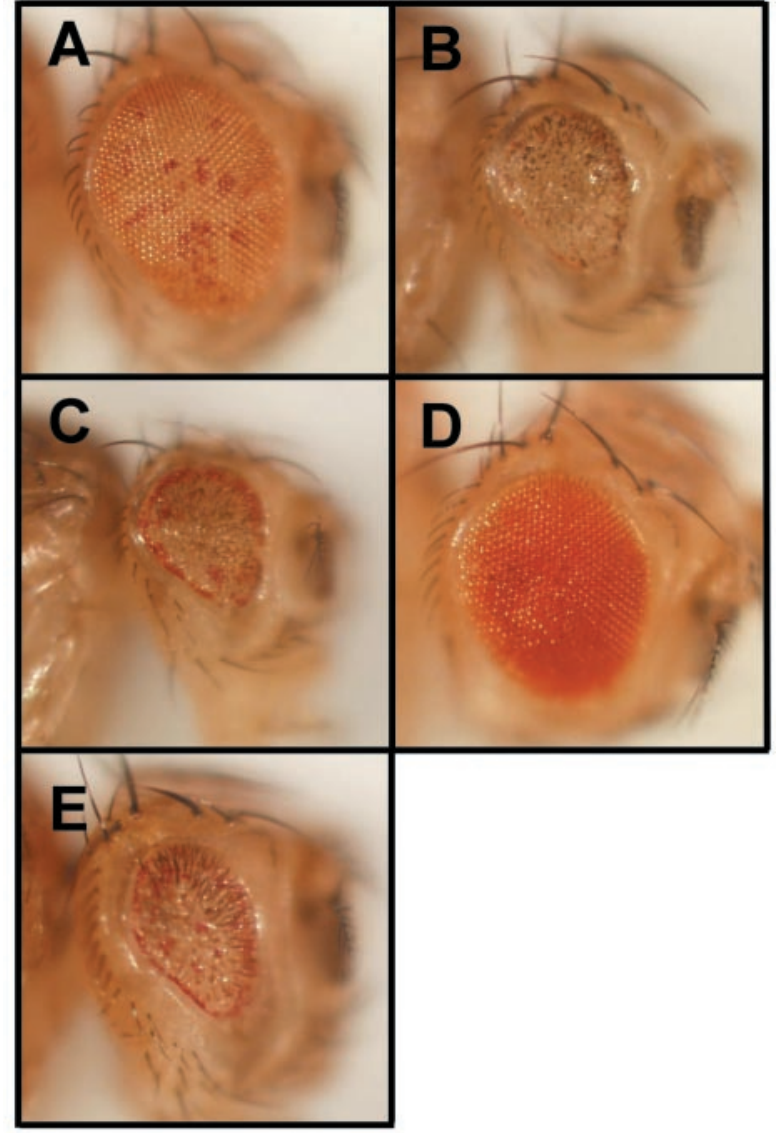

FIG. 5. DRONC overexpression phenotypes in different genetic backgrounds. The following genotypes are shown: GMRDRONC $^{\mathrm{M}} /+(A)$; GMR-DRONC $/+(B)$; GMR-DRONC $\mathrm{M} /+$ and $t h 5 /+$ $(C)$; GMR-DRONC $/+$ and GMR-DIAP1/+ $(D)$; GMR-DRONC $/+$ and GMR-p35 $(E)$. Expression of intermediate levels of DRONC in the eye $\left(\right.$ GMR-DRONC $\left.{ }^{\mathrm{M}}\right)$ resulted in flies with normal sized eyes, but in which pigment was largely absent $(A)$. High level expression of DRONC in the fly eye (GMR-DRONC ${ }^{\mathrm{S}}$ ) resulted in flies with small eyes $(B)$. GMRDRONC ${ }^{\mathrm{M}}$ flies that were heterozygous for $t h 5$, a mutation in DIAP1, had eyes that were smaller than those of GMR-DRONC ${ }^{\mathrm{M}}$ flies $(C)$. Expression of GMR-DIAP1 suppressed the GMR-DRONC ${ }^{\mathrm{S}}$ small eye phenotype $(D)$. Expression of GMR-p35 did not significantly suppress the GMR-DRONC ${ }^{\mathrm{S}}$ small eye phenotype $(E)$.

size and shape, but that were largely white due to a loss of retinal pigment (Fig. 5A). Tangential sections through the eyes of GMR-DRONC ${ }^{\mathrm{M}}$ flies showed that all retinal cells, including photoreceptors, were missing (data not shown). Increasing DRONC expression levels still further (GMR-DRONC ${ }^{\mathrm{S}}$ flies) resulted in flies with small eyes (Fig. $5 B$ ), similar to those seen in animals overexpressing $r p r$, hid, or grim. These observations, similar to those described by Meier et al. (28), show that DRONC expression in the eye induces cell death in a dose-dependent manner. Consistent with this interpretation, third instar eye imaginal discs from animals expressing GMRDRONC $^{\mathrm{S}}$ showed high levels of staining with the vital dye acridine orange (data not shown), which is taken up and retained by dying cells (50).

DIAP1, a Drosophila member of the IAP family of caspase inhibitors, suppresses rpr-, hid-, and grim-dependent cell death in the fly (reviewed in Ref. 21). ${ }^{2}$ We reasoned that if expression of DRONC was activating the same pathway, then the GMRDRONC eye phenotype might be sensitive to the levels of DIAP1. To test this hypothesis we decreased the amount of DIAP1 in the eye by crossing a strong loss-of-function DIAP1

${ }^{2}$ S. J. Yoo and C. J. Hawkins, unpublished data 
A

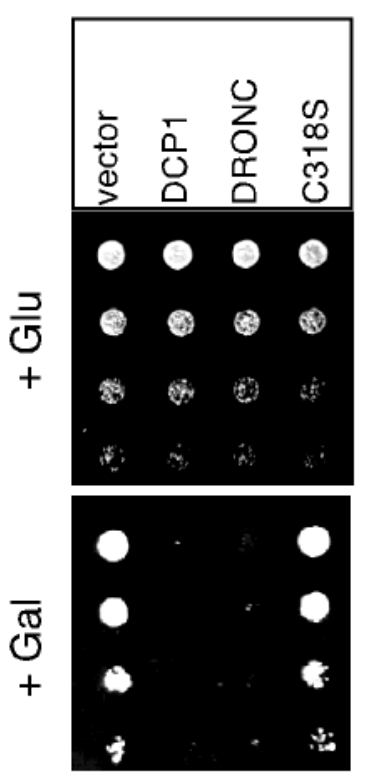

C

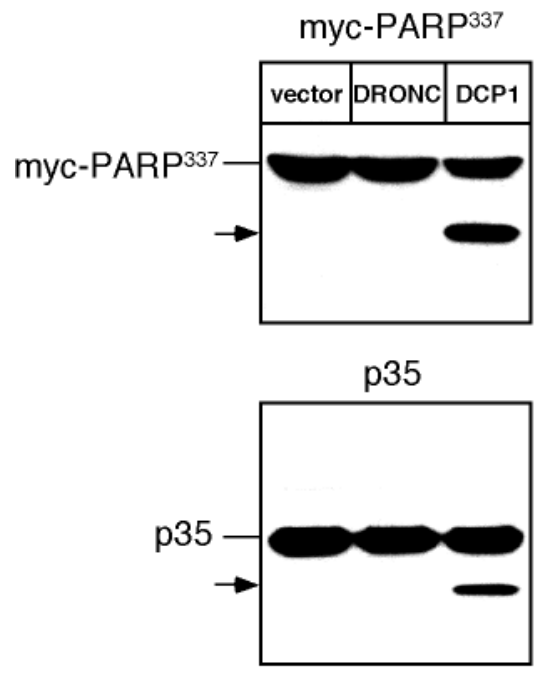

B
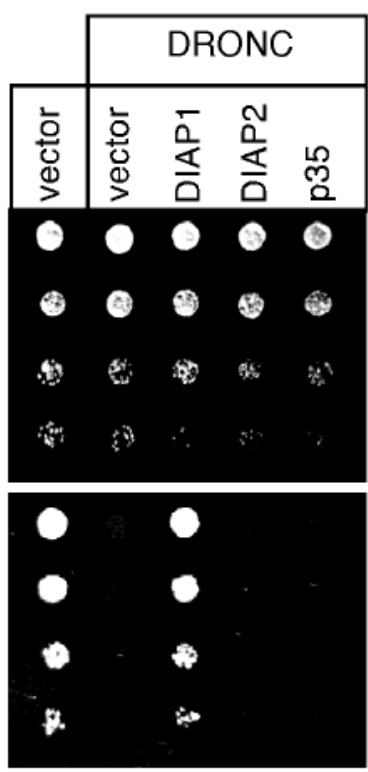

D

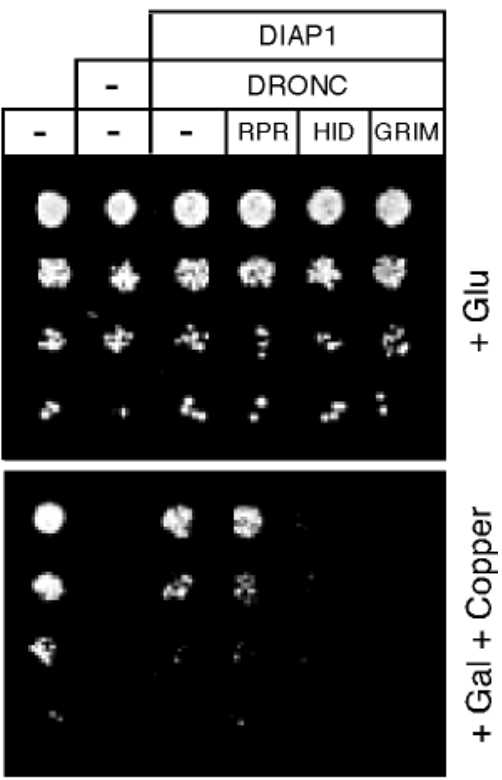

point mutant, thread 5 (th5), to GMR-DRONC ${ }^{\mathrm{M}}$ flies. th 5 heterozygotes are phenotypically wild type. However, flies that were heterozygous for $t h 5$, and that expressed GMR-DRONC ${ }^{\mathrm{M}}$, showed an enhancement of the GMR-DRONC-dependent small eye phenotype (Fig. $5 C$ ). In contrast, small eyed GMR-DRONC ${ }^{\mathrm{S}}$ flies that overexpressed DIAP1 because they carry a GMRDIAP1 transgene showed a strong suppression of the small eye and pigment loss phenotypes (Fig. $5 D$ ). These observations, and similar observations made by Meier et al. (28), are consistent with the idea that DRONC activity is negatively regulated by DIAP1. However, they do not exclude the possibility that DIAP1s effects on the DRONC overexpression phenotypes are due, at least in part, to DIAP1s ability to suppress the activity of caspases such as drICE (33), that are activated by DRONC. Interestingly, the $\mathrm{DRONC}^{\mathrm{S}}$ eye phenotype was not suppressed by coexpression of the baculovirus caspase inhibitor p35 (Fig. 5E) (28). This result was surprising because p35 is generally thought of as a broad specificity caspase inhibitor (reviewed in Ref. 51), and because expression of GMRDRONC $^{\text {C318S }}$ (Ref 28, this work) or GMR-p35 (23-26), suppressed rpr-, hid-, and grim-dependent cell death in the fly eye.

To characterize DRONCs interactions with caspase inhibitors further, in isolation from other Drosophila cell death regulators, we made use of a yeast caspase activity reporter system in which caspase activity is monitored as a function of cell survival. In this system, high level expression of active caspases kills yeast, and this death is suppressed by coexpression of proteins that inhibit caspase activity $(39,52)$. Yeast were transformed with a galactose-inducible expression vector (carrying the GAL1 promoter) that was either an empty vector, or that contained wild type DCP-1, wild type DRONC, or the 
catalytically inactive DRONC ${ }^{\mathrm{C} 318 \mathrm{~S}}$. Transformants were spotted as a series of 5 -fold serial dilutions onto glucose plates to indicate the number of cells, and onto galactose plates to induce expression of the caspase. As shown in Fig. 6A, cells expressing the empty vector grew well on glucose or galactose-containing media. However, cells expressing GAL1-DCP-1 or GAL1-DRONC failed to grow on galactose-containing media. Importantly, cells expressing GAL1-DRONC ${ }^{\mathrm{C} 318 \mathrm{~S}}$ grew on galactose as well as cells expressing the empty vector. Thus, DRONC expression kills yeast and this killing requires caspase activity.

To determine if DRONC activity could be inhibited by known or suspected caspase inhibitors we carried out experiments in which GAL1-DRONC expression was induced in cells carrying a second vector in which expression was driven by the constitutive ADH1 promoter. This vector was either empty, or carried the coding region for DIAP1, a second cell death inhibiting Drosophila IAP, DIAP2 (reviewed in Ref. 21), or baculovirus p35. As shown in Fig. 6B, expression of ADH-DIAP1 blocked DRONC-dependent cell death, but expression of ADH-DIAP2 or ADH-p35 did not. DIAP2 interacts with RPR, HID, and GRIM $(53,54)$, and blocks rpr-, hid-, and grim-dependent cell death $(24,54-56)$. However, interactions between DIAP2 and lepidopteran or Drosophila caspases have not been observed (57). ${ }^{3}$ DIAP2 may suppress death by interacting with unidentified caspases; alternatively DIAP2 may function primarily to inhibit the activity of caspase-activating proteins such as RPR, HID, and GRIM. Inhibition of caspase activity by p35 is associated with p35 cleavage, resulting in the formation of a stable p35-caspase complex $(58,59)$. Because of DRONC's unique cleavage specificity we asked if DRONC was in fact able to cleave p35. Yeast were transformed with an ADH1 expression plasmid carrying the coding region for a Myc- tagged fragment of a common caspase substrate, poly(ADP-ribosyl)transferase (PARP) (reviewed in Ref. 60), or baculovirus p35. These yeast also carried GAL1 vectors that were either empty, or that contained DRONC or DCP-1. Extracts were made from yeast grown under GAL1 inducing conditions and these were processed for SDS-PAGE and Western blotting using anti-Myc or p35 antibodies. As shown in Fig. 6C, only the full-length substrate band was seen in yeast expressing Myc-PARP or p35 in isolation or in combination with DRONC. In contrast, yeast expressing Myc-PARP or p35 in combination with DCP-1 showed immunoreactive bands of sizes expected following caspase cleavage. These results and those of Meier et al. (28) further support the idea that DRONC has a unique cleavage specificity, and strongly suggest that DRONC-dependent cell killing is not inhibited by p35 because DRONC does not recognize and/or cleave the caspase target site in p35.

We found that bacterially produced DIAP1 did not inhibit the activity of fully processed (prodomainless) DRONC in vitro (data not shown). This stands in contrast to our observation that DIAP1 inhibited DRONC-dependent yeast cell death. These two sets of observations are not necessarily incompatible. The DRONC prodomain has been shown to bind to DIAP1 (28). Also the small eye phenotypes associated with overexpression of full-length DRONC (Ref. 28 and this work), but not versions of DRONC lacking the prodomain (28), are sensitive to the levels of DIAP1. These observations suggest that effective DIAP1 inhibition of DRONC activity requires the DRONC prodomain (28). DIAP1 may, for example, block a step of DRONC processing, and thus DRONC activation. Alternatively, DIAP1 may block the activity of partially processed, prodomain containing, versions of DRONC. Other observations pointing toward an important role for the prodomain in some

${ }^{3}$ C. J. Hawkins and S. J. Yoo, unpublished observations.
DIAP1-caspase interactions comes from the finding that drICE lacking its prodomain was also not inhibited by DIAP1 (39), while versions containing the prodomain were (33). We were unable to generate an active version of DRONC containing its prodomain (DRONC ${ }^{\mathrm{pD} 4 \mathrm{~A}}$ ), and have thus been unable to directly test the hypothesis that DIAP1 inhibits DRONC activation or activity. Nonetheless, the observations that DIAP1 physically interacts with the DRONC prodomain, that DRONCdependent death is sensitive to the levels of DIAP1 in flies and in yeast, and that there is a good correlation between IAP-dependent inhibition of caspase-dependent phenotypes in yeast and caspase activity in vitro $(33,39)$, argue that this is likely to be the case.

HID and GRIM Suppress DIAP1s Ability to Inhibit DRONCdependent Yeast Cell Death-Genetic and biochemical evidence suggests that one mechanism by which RPR, HID, and GRIM promote apoptosis is by blocking DIAP1s ability to inhibit caspase activation or activity, thereby promoting caspase-dependent cell death $(33,34,57)$. To determine if DRONCs activity could be regulated in a similar manner we tested whether RPR, HID, or GRIM could interfere with DIAP1-dependent inhibition of DRONC-dependent yeast cell death. We generated yeast in which DRONC was expressed under GAL1 control and DIAP1 was expressed under the control of the copper-inducible CUP1 promoter. We then introduced into these yeast a third GAL1 vector that was either empty or that expressed RPR, HID, or GRIM. As shown in Fig. 6D, cells expressing GAL1DRONC and empty vectors died when plated on medium containing galactose and $100 \mu \mathrm{M}$ copper, but cells expressing GAL1-DRONC and CUP1-DIAP1 survived. Coexpression of GAL1-RPR had no effect on the survival of yeast expressing GAL1-DRONC and CUP1-DIAP1. However, coexpression of GAL1-HID or GAL1-GRIM completely blocked the survival of these cells (Fig. 6D). RPR, HID, or GRIM expression in isolation has little or no effect on yeast cell growth (33). Thus, while these experiments do not exclude the possibility that HID and GRIM might alter DRONC activity directly, they are consistent with other observations arguing that these proteins mediate their effects on caspase activity, and thus presumably caspasedependent yeast cell killing, by virtue of their interactions with DIAP1 $(33,34)$.

We cannot formally exclude the possibility that DRONC normally functions to regulate cellular processes other than cell death since mutants that eliminate DRONC are not available. However, the observations by ourselves and others (28) demonstrating that dominant negative forms of DRONC block rpr-, hid-, and grim-dependent cell death, that DRONC kills, and that DRONC interacts with drICE, DIAP1, HID, and GRIM, all support the idea that DRONC plays an important role in promoting apoptotic cell death. However, DRONC activity is not inhibited by p35, which is a potent inhibitor of rpr-, hid-, and grim-dependent cell death in the fly. Interestingly, an analogous p35-insensitive, IAP-sensitive, cell death-activating cleavage event has also been described in lepidopteran cells. Baculovirus infection of lepidopteran cells elicits a cell death response that is associated with cleavage and activation of the lepidopteran caspase Sf caspase-1 (61-63). This cleavage is blocked by viral IAPs, but not by p35. Instead, p35-dependent death suppression in this system is associated with inhibition of active Sf caspase- $1(62,63)$. Sf caspase- 1 cleavage is probably mediated by $\mathrm{p} 35$-insensitive proteases and not by oligomerization followed by autocatalysis (63). This is because the Sf caspase-1 prodomain is short and lacks death effector domain or caspase recruitment domain sequences that might mediate the oligomerization required for this mode of activation (64). DRONC-dependent killing of mammalian cells was blocked by 
p35 (18). However, based on our observation that DRONC cleaves the executioner caspase drICE at the same site as the more typical caspase DCP-1, as well as the above noted findings in lepidopteran cells, it seems likely that p35 blocked DRONC-dependent killing of mammalian cells by inhibiting the activity of caspases activated by DRONC. Further indirect evidence supporting a possible role for a caspase such as DRONC in apoptosis initiation, that is p35-insensitive and has a novel cleavage specificity, comes from the observation that cleavage of DCP-2/DREDD, which occurs following overexpression of RPR, HID, or GRIM, is p35-insensitive (17). Finally, it is worth noting that the caspase DECAY lacks aspartate residues in the region predicted to span the large-small subunit boundary (Fig. 1) (19). This indicates that processing of this caspase, if it occurs, must happen at other residues.

Models for how DRONC might function as a death activator must explain how DRONC-dependent death can be sensitive to p35, while DRONC itself is p35-insensitive. In considering DRONC-dependent cell death it is important to recognize that the cell death seen when DRONC is expressed in the eye under the control of the GMR promoter may be the result of unphysiologically high levels of DRONC activity, which lead to cleavage of inappropriate substrates and cell death. This DRONCdependent death would be expected to be sensitive to the levels of DIAP1 because DIAP1 inhibits DRONC activity, but insensitive to p35 because DRONC does not interact with p35 (see Figs. 5 and 6). drICE is p35-inhibitable and DRONC processes drICE between the large and small subunits, presumably activating it. However, DRONC does not process the drICE prodomain. Versions of drICE that contain prodomain sequences are inhibited by DIAP1 (33), but drICE lacking its prodomain is DIAP1-insensitive (39). Thus, the normal levels of DIAP1 present in the eye may act as a buffer to limit the activity of drICE that has been processed by DRONC alone. This would also contribute to making the DRONC overexpression phenotype p35-insensitive. In this and as discussed below we refer specifically to drICE as a target of DRONC and as a likely executioner caspase based on the observations presented here and elsewhere $(15,28,30,32)$. However, other executioner caspases may be important and behave similarly.

There are several ways in which DRONC could play an important role in initiating p35-sensitive cell death. One possibility is simply that the levels of DRONC activity required to activate p35-inhibitable executioner caspases are low enough that DRONC activation in isolation does not induce apoptosis. A second possibility is that upstream death signals lead to activation of DRONC as well as other caspases with more traditional cleavage specificities. In this model DRONC and the other initiator caspases both cleave and activate executioner caspases such as drICE. If any of these caspases process drICE in the prodomain (as DCP-1 does (Fig. 3A)) this would promote the formation of a DIAP1-insensitive form of drICE, thereby presumably amplifying drICE activity. In this scenario, as above, it is proposed that the levels of DRONC activity are low compared with those present during DRONC overexpression. Therefore p35 should be able to block cell death since p35 is able to inhibit drICE-activating caspases with traditional caspase cleavage sites, as well as drICE itself. Finally, upstream death signals leading to activation of DRONC may result in the inhibition of caspase inhibitors such as DIAP1 as a consequence of its interacting with molecules such as RPR, HID, or GRIM (33). In this model, DRONC activation of executioner caspases such as drICE, coupled with a decrease in DIAP1 function, would lead to increased activation of drICE, which is p35 inhibitable. As above, this model assumes that the levels of DRONC activity induced by upstream cell death sig- nals are not sufficient to promote significant cell death in isolation. Clearly these models are not mutually exclusive. An important test of each of these models will be to determine if DRONC activation occurs following rpr, hid, or grim overexpression, even if cell death is blocked by coexpression of p35.

Several caspases, including Drosophila DRONC and C. elegans CSP-1 and CSP-2, have divergent active site pentapeptide sequences. We showed that one of these, DRONC, has a novel cleavage site specificity, cleaving $\mathrm{COOH}$-terminal to glutamate as well as aspartate residues. Our observations with DRONC are important because they suggest that some caspasedependent processes have remained unseen using caspase inhibitors such as p35. It will be interesting to see if cell deaths, or steps in cell death signaling that have been thought to be caspase-independent, and thus not amenable to caspase-based therapeutics, are in some cases regulated by caspases with nontraditional cleavage specificities.

Acknowledgments-We thank Becky Green-Maruoquin for sectioning Drosophila eyes and Gary M. Hathaway for microsequencing.

\section{REFERENCES}

1. Jacobson, M. D., Weil, M., and Raff, M. C. (1997) Cell 88, 347-354

2. Alnemri, E. S., Livingston, D. J., Nicholson, D. W., Salvesen, G. S., Thornberry, N. A., Wong, W. W., and Yuan, J. (1996) Cell 87, 171

3. Walker, N. P. C., Talanian, R. V., Brady, K. D., Dang, L. C., Bump, N. J., Ferenz, C. R., Franklin, S., Ghayur, T., Hackett, M. C., Hammill, L. D., and et al. (1994) Cell 78, 343-352

4. Wilson, K. P., Black, J. A. F., Thomson, J. A., Kim, E. E., Griffith, J. P., Navia, M. A., Murcko, M. A., Chambers, S. P., Aldape, R. A., Raybuck, S. A., and Livingston, D. J. (1994) Nature 370, 270-275

5. Rotonda, J., Nicholson, D. W., Fazil, K. M., Gallant, M., Gareau, Y., Labelle, M., Peterson, E. P., Rasper, D. M., Ruel, R., Vaillancourt, J. P., Thornberry, N. A., and Becker, J. W. (1996) Nat. Struct. Biol. 3, 619-625

6. Mittl, P. R., Di Marco, S., Krebs, J. F., Bai, X., Karanewsky, D. S., Priestle, J. P., Tomaselli, K. J., and Grutter, M. G. (1997) J. Biol. Chem. 272, 6539-6547

7. Sleath, P. R., Hendrickson, R. C., Kronheim, S. R., March, C. J., and Black, R. A. (1990) J. Biol. Chem. 265, 14526-14528

8. Howard, A. D., Kostura, M. J., Thornberry, N., Ding, G. J., Limjuco, G., Weidner, J., Salley, J. P., Hogquist, K. A., Chaplin, D. D., Mumford, R. A., Schmidt, J. A., and Tocci, M. J. (1991) J. Immunol. 147, 2964-2969

9. Thornberry, N. A., Bull, H. G., Calaycay, J. R., Chapman, K. T., Howard, A. D. Kostura, M. J., Miller, D. K., Molineaux, S. M., Weidner, J. R., Aunins, J., and et al. (1992) Nature 356, 768-774

10. Thornberry, N. A., Rano, T. A., Peterson, E. P., Rasper, D. M., Timkey, T., Garcia-Calvo, M., Houtzager, V. M., Nordstrom, P. A., Roy, S., Vaillancourt, J. P., Chapman, K. T., and Nicholson, D. W. (1997) J. Biol. Chem. 272, 17907-17911

11. Talanian, R. V., Quinlan, C., Trautz, S., Hackett, M. C., Mankovich, J. A., Banach, D., Ghayur, T., Brady, K. D., and Wong, W. W. (1997) J. Biol. Chem. 272, 9677-9682

12. Salvesen, G. S., and Dixit, V. M. (1997) Cell 91, 443-446

13. Cohen, G. M. (1997) Biochem. J. 326, 1-16

14. Song, Z., McCall, K., and Steller, H. (1997) Science 275, 536-540

15. Fraser, A. G., and Evan, G. I. (1997) EMBO J. 16, 2805-2813

16. Inohara, N., Koseki, T., Hu, Y., Chen, S., and Nunez, G. (1997) Proc. Natl. Acad. Sci. U. S. A. 94, 10717-10722

17. Chen, P., Rodriguez, A., Erskine, R., Thach, T., and Abrams, J. M. (1998) Dev. Biol. 201, 202-216

18. Dorstyn, L., Colussi, P. A., Quinn, L. M., Richardson, H., and Kumar, S. (1999) Proc. Natl. Acad. Sci. U. S. A. 96, 4307-4312

19. Dorstyn, L., Read, S. H., Quinn, L. M., Richardson, H., and Kumar, S. (1999) J. Biol. Chem. 274, 30778-30783

20. Rubin, G. M., Yandell, M. D., Wortman, J. R., Miklos, G. L. G., Nelson, C. R., Hariharan, I. K., and et al. (2000) Science 287, 2204-2215

21. Bergmann, A., Agapite, J., and Steller, H. (1998) Oncogene 17, 3215-3223

22. Hay, B. A., Wolff, T., and Rubin, G. M. (1994) Development 120, 2121-2129

23. Grether, M. E., Abrams, J. M., Agapite, J., White, K., and Steller, H. (1995) Genes Dev. 9, 1694-1708

24. Hay, B. A., Wassarman, D. A., and Rubin, G. M. (1995) Cell 83, 1253-1262

25. White, K., Tahaoglu, E., and Steller, H. (1996) Science 271, 805-807

26. Chen, P., Nordstrom, W., Gish, B., and Abrams, J. M. (1996) Genes Dev. 10, 1773-1782

27. Rodriguez, A., Oliver, H., Zou, H., Chen, P., Wang, X., and Abrams, J. M. (1999) Nat. Cell Biol. 1, 272-279

28. Meier, P., Silke, J., Leevers, S. J., and Evan, G. I. (2000) EMBO J. 19, 598-611

29. McCall, K., and Steller, H. (1998) Science 279, 230-234

30. Kanuka, H., Sawamoto, K., Inohara, N., Matsuno, K., Okano, H., and Miura M. (1999) Mol. Cell 4, 757-769

31. Zhou, L., Song, Z., Tittel, J., and Steller, H. (1999) Mol. Cell 4, 745-755

32. Fraser, A. G., McCarthy, N. J., and Evan, G. I. (1997) EMBO J. 16, 6192-6199

33. Wang, S. L., Hawkins, C. J., Yoo, S. J., Muller, H. A., and Hay, B. A. (1999) Cell 98, 453-463

34. Goyal, L., McCall, K., Agapite, J., Hartwieg, E., and Steller, H. (2000) EMBO J. 19, $589-597$ 
35. Lisi, S., Mazzon, L., and White, W. (2000) Genetics 154, 669-678

36. Thornberry, N. A., and Lazebnik, Y. (1998) Science 281, 1312-1316

37. Shaham, S. (1998) J. Biol. Chem. 273, 35109-35117

38. Rano, T. A., Timkey, T., Peterson, E. P., Rotunda, J., Nicholson, D. W., Becker, J. W., Chapman, K. T., and Thornberry, N. A. (1997) Chem. Biol. 4, 149-155

39. Hawkins, C. J., Wang, S. L., and Hay, B. A. (1999) Proc. Natl. Acad. Sci. U. S. A. 96, 2885-2890

40. Garcia-Calvo, M., Peterson, E. P., Rasper, D. M., Vaillancourt, J. P., Zamboni, R., Nicholson, D. W., and Thornberry, N. A. (1999) Cell Death Diff. 6, 362-369

41. Stennicke, H. R., and Salvesen, G. S. (1999) Methods 17, 313-319

42. Hawkins, C. J., Uren, A. G., Hacker, G., Medcalf, R. L., and Vaux, D. L. (1996) Proc. Natl. Acad. Sci. U. S. A. 93, 13786-13790

43. Spradling, A. C., and Rubin, G. M. (1982) Science 218, 341-347

44. Wolff, T., and Ready, D. F. (1991) Development 113, 825-839

45. Song, Z., Bergmann, A., Nicholson, D. W., Thornberry, N. A., Peterson, E. P., and Steller, H. (2000) Mol. Cell. Biol. 20, 2907-2914

46. Rodriguez, J., and Lazebnik, Y. (1999) Genes Dev. 13, 3179-3184

47. Saleh, A., Srinivasula, S. M., Acharya, S., Fishel, R., and Alnemri, E. S. (1999) J. Biol. Chem. 274, 17941-17945

48. Zou, H., Li, Y., Liu, X., and Wang, X. (1999) J. Biol. Chem. 274, 11549-11556

49. Spradling, A. C., and Rubin, G. M. (1983) Cell 34, 47-57

50. Spreij, T. E. (1971) Netherlands J. Zool. 21, 221-264

51. Graczyk, P. P. (1999) Restor. Neurol. Neurosci. 14, 1-23
52. Kang, J. J., Schaber, M. D., Srinivasula, S. M., Alnemri, E. S., Litwack, G., Hall, D. J., and Bjornsti, M. A. (1999) J. Biol. Chem. 274, 3189-3198

53. Vucic, D., Kaiser, W. J., Harvey, A. J., and Miller, L. K. (1997) Proc. Natl. Acad. Sci. U. S. A. 94, 10183-10188

54. Vucic, D., Kaiser, W. J., and Miller, L. K. (1998) Mol. Cell. Biol. 18, 3300-3309 55. Vucic, D., Seshagiri, S., and Miller, L. K. (1997) Mol. Cell. Biol. 17, 667-676

56. Wing, J. P., Zhou, L., Schwartz, L. M., and Nambu, J. R. (1998) Cell Death Differ. 5, 930-939

57. Kaiser, W. J., Vucic, D., and Miller, L. K. (1998) FEBS Lett. 440, 243-248

58. Bump, N. J., Hackett, M., Hugunin, M., Seshagiri, S., Brady, K., Chen, P. Ferenz, C., Franklin, S., Ghayur, T., Li, P., Licari, P., Mankovich, J., Shi, L. F., Greenberg, A. H., Miller, L. K., and Wong, W. W. (1995) Science 269, 1885-1888

59. Xue, D., and Horvitz, H. R. (1995) Nature 377, 248-251

60. Cryns, V., and Yuan, J. (1998) Genes Dev. 12, 1551-1570

61. Manji, G. A., Hozak, R. R., LaCount, D. J., and Friesen, P. D. (1997) J. Virol. 71, 4509-4516

62. Seshagiri, S., and Miller, L. K. (1997) Proc. Natl. Acad. Sci. U. S. A. 94, 13606-13011

63. LaCount, D. J., Hanson, S. F., Schneider, C. L., and Friesen, P. D. (2000) J. Biol. Chem. 275, 15657-15664

64. Ahmad, M., Srinivasula, S. M., Wang, L., Litwack, G., Fernandes-Alnemri, T., and Alnemri, E. S. (1997) J. Biol. Chem. 272, 1421-1424 